\title{
Building a Corpus of Facial Expressions for Learning-Centered Emotions
}

\author{
María Lucía Barrón-Estrada, Ramón Zatarain-Cabada, \\ Bianca Giovanna Aispuro-Medina, Elvia Minerva Valencia-Rodríguez, \\ Ana Cecilia Lara-Barrera \\ Instituto Tecnológico de Culiacán, Culiacán, Sinaloa, \\ Mexico \\ \{lbarron, rzatarain, m06170904,m95170906,m15171452\} @itculiacan.edu.mx
}

\begin{abstract}
Recognizing emotions in software interfaces is very important today and even more in the educational field where the students can, through their emotions, reveal affective states related or not to the learning process. An educational software with the capability to recognize emotions will allow the students to receive appropriate feedback to their personal cognitive needs. In this paper a method is presented for the construction of an image database of facial expressions, in which, through the detection of EGG signals corresponding to affective states focused on education (engagement, bored, frustrated and meditation), detects and registers spontaneous facial expressions. We also show experiments with the obtained results.
\end{abstract}

Keywords: Face expression recognition, affective computing, EEG recognition, face expression database.

\section{$1 \quad$ Introduction}

The emotional state of a person is important because it enables or restricts the performance of its actions to achieve different goals. The study of emotions and the affective states had become increasingly popular over the last 20 years including them on the computing systems that we use nowadays. In the educational area, the emotions of the individuals are transcendental to the learning process. Several research works $[1,2,3]$ had been developed to study the existing relation between the affective state of the student and the cognitive processes through the use of intelligent systems of learning. Some of the most important affective states focused on education are bored, confused, frustrated and engagement [4].

The purpose of this work was to build an image database of spontaneous facial expressions that corresponds to affective states focused on education. The main purpose was to use the corpus in different Intelligent Tutoring Systems (ITS). The main motivation of this work emerges from the need of having an image database of spontaneous facial expressions corresponding to affective states focused on education, since the existent data bases have issues such as: they only contain 
images from people with posed facial expressions to represent an emotion and the images are from people with physiological features different from our community.

For the creation of the corpus, we needed to capture the images and label them with the emotion that they represent. The Emotiv EPOC headset, was used to perform the reading of EGG signals in persons participating in the experiment; this device allows to detect affective states focused on education using the affective suite. Additionally, to capture the image of the facial expression corresponding to the affective state of the person, a camera C920 Logitech HD Pro Webcam was used.

This paper is organized as follows: in the second section the related work is described; a description of the technology used to build the corpus is presented in the third section; in the fourth section the methodology for the development of the corpus is shown; the experiment and the obtained results are described in the fifth section; finally in the sixth section we present the conclusions.

\section{$2 \quad$ Related Work}

In this section some important works related to databases of facial expressions are presented and discussed.

Radboud Faces Database (RaFD) is a database that contains photographs of a group of 67 models, formed by Caucasians adults and children. Each model was trained to show 8 different facial expressions (anger, disgust, fear, happiness, sadness, contempt and neutral) and each emotion is shown with three different gaze directions where all photographs were taken from 5 different camera angles simultaneously in a highly controlled environment [5].

JAFFE Database is a database of facial expressions acted by Japanese women. The database contains facial expressions of over 60 women and each one of them presents 3 to 4 expressions of the following emotions: neutral, happy, anger, disgust, fear, sadness and surprise. The database contains a total of 219 images on grey scale [5]. In figure 2 two participants can be observed showing the seven expressions previously mentioned.

Database for Emotion Analysis using Physiological Signals (DEAP), is a multimodal database which presents a data set for the analysis of human affective states based on the basic emotions proposed by Ekman (fear, anger, happy, sadness, disgust and surprise). In this database EGG and physiological signals of 32 participants were recorded as each watched 40 one-minute long excerpts of music videos. Participants rated each video in terms of levels of arousal, valence, like/dislike, dominance and familiarity. For 22 of the 32 participants frontal face video was also recorded. The correlation between EGG signals and the participant ratings was investigated. The data set is publicly available so it can be used on investigations regarding estimation methods of affective states [6].

NVIE Database is a Natural Visible and Infrared Facial Expression Database that contains both posed and spontaneous expressions performed by 215 students (157 men and 58 women). The evaluated emotions were: happiness, sadness, surprise, fear, anger and disgust. The spontaneous emotions were induced by 
screening deliberately emotional videos. Besides of capturing the facial expressions the goal was to analyze the relation between facial temperature and the emotion through a statistical analysis [7].

Cohn-Kanade dataset is a database that contains images of 100 college students between 18 and 35 years old. From this population, 65\% are women, 15\% are Africa-Americans, and only 3\% are Asian or Spanish. All of the subjects were recorded acting a total of 23 facial expressions corresponding to seven emotions (neutral, anger, disgust, fear, joy, sadness and surprise) [8] using a video camera. This database was released in order to promote investigation on the individual automatic recognition of facial expressions, since then it has become the database of facial expressions more used in the development of algorithms and assessments of this kind [9].

The database of facial expressions that is presented in this paper distinguishes itself from the previous mentioned works in that the registered emotions are focused specifically in the learning process.

\section{Technology}

Emotiv EPOC is a brain-computer interface, created by Emotiv Systems. It is used in researching applications and contains three components named "suites" to process the signals: Expressive suite, Cognitive suite, and Affective suite.
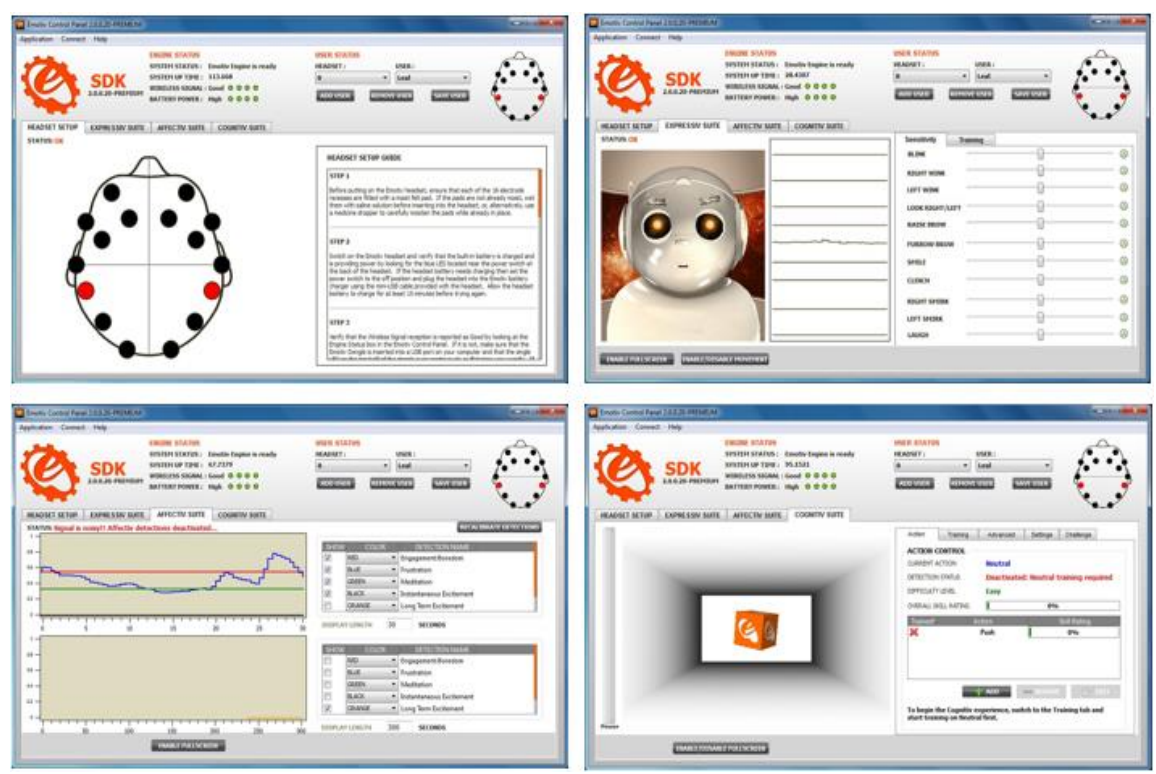

Fig. 1. Emotiv EPOC suite's interfaces.

The expressive Suite determines the facial expressions in real time according to the signals received by the interface. An avatar appears in the computer's screen 
imitating the user's facial expressions. This action make possible a natural interaction. The cognitive suite interprets the user's thinking and intentions. The affective suite monitors the user's affective states in real time. Figure 1 shows the Emotiv interfaces.

The Emotiv EPOC headset has two support electrodes. They are set behind each ear in the skull's protuberant zone and are considered as points of reference to guide the correct placement of the rest of the electrodes. With the Control panel included in the kit, it is possible a graphic visualization of the electrodes' status to be able to know if the electrodes are positioned correctly or the electrodes need an adjustment. There is also the TestBench application to visualize the EGG signals in real time.

\section{Application Development to Build the Database}

The affective states detected by Emotiv EPOC considered in the creation of the facial expressions database are: engagement, boring, frustration and meditation. A Java application was implemented in order to create the facial image database considering the affective state when using the Emotiv headset. Emocomposer was required to emulate the headset operation. Figure 2 shows the environment and components of the application. The environment of the application consist of different components around the Emotiv's SDK and the Emotiv EPOC headset. The Java program running in the PC was implemented with the NetBeans IDE development environment. A jna.jar library was needed to make possible the interaction of the application and Emotiv EPOC headset. The open source OpenCV library was incorporated to perform the capture of the images using a Web Cam.

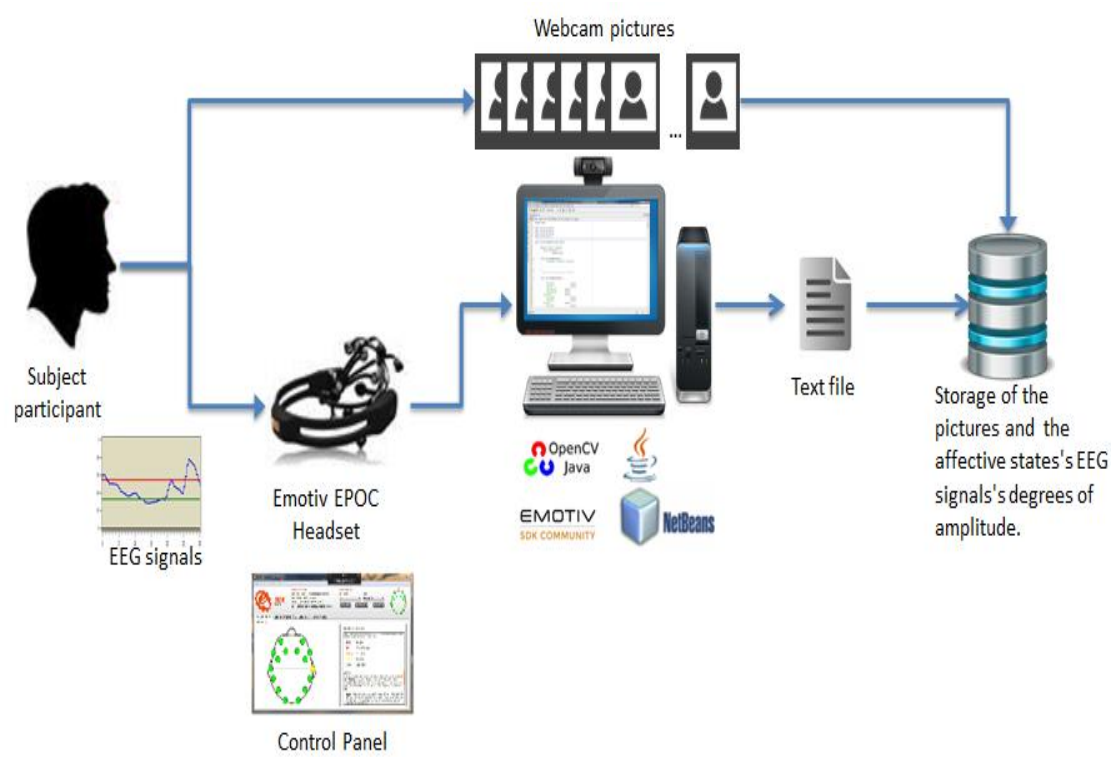

Fig. 2. Environment to create the facial expression database. 


\subsection{Testing the Environment}

Some situations were detected and solved: lighting troubles which were solved using two extra white light lamps in addition to the experimentation room illumination. Figure 3 shows the general experiment environment, where the participant, as can be appreciated, is in front of the monitor, the position of the illumination lamps, a web cam, and the Emotiv EPOC headset.

\subsection{Important Points in the Final Experiment}

In the Final Experiment phase, the captured images were stored to feed the facial expressions database, using a partially controlled environment in which aspects like illumination, background, and acoustic were considered, to allow the participant express his/her emotions in a natural manner. Next, each experiment phase is described.

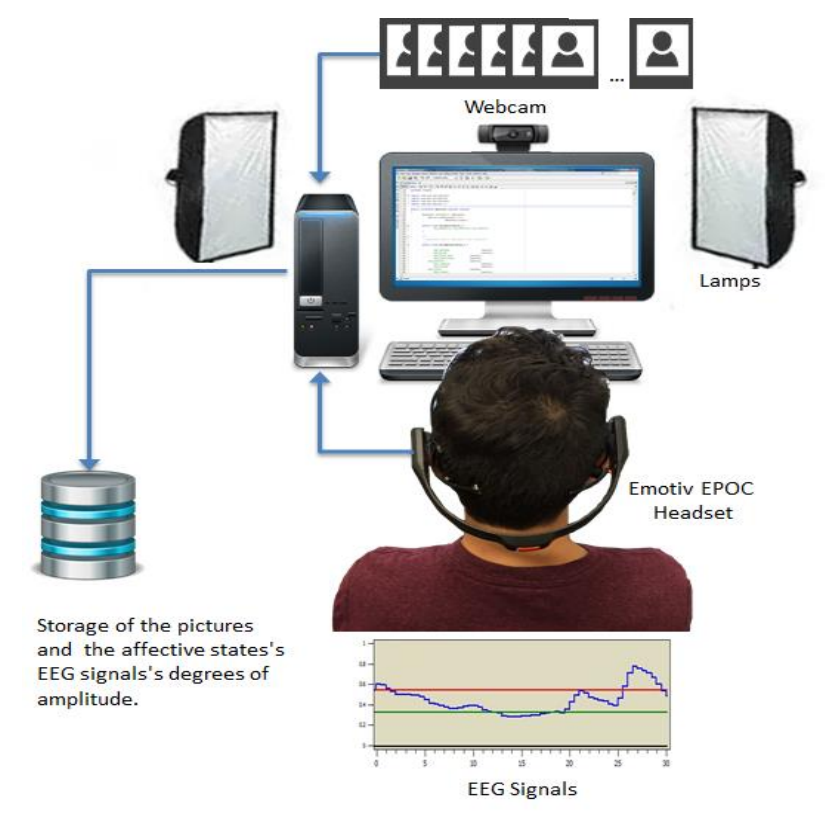

Fig. 3. Experiment environment.

A group of Master degree students from Instituto Tecnológico de Culiacán was chosen to participate in the final experiment. It was conformed of 8 students ( 5 men and 3 women), ages from 24 to 47 . Intending to homologate the initial states of the subjects, they were instructed to follow the next recommendations: Sleep at least 8 hours the day before the experiment, had a good breakfast, do not consume coffee or energy drinks, do not consume substances that affect the nervous system, do not use gel or hair spray the day of experiment, do not wear glasses and wear dark clothes the day of the experiment. 
The materials used in the experiment were: The Emotiv SDK Kit; a Laptop with Windows 7 Professional 64-bit operating system, Intel (R) Core (TM) i3-2310M and 4.00 GB RAM; the development environment NetBeans 8.1; Open CV library to capture the images; Java platform: Standard Edition Development Kit (JDK TM) to 32 bits; Logitech Webcam C920 HD Pro; Java application EmoStateLog Emotiv SDK and application implemented for the experiment; the Emotiv EPOC headset.

Some examples of programming exercises on Java that were provided to the subject participant to perform cognitive activities for 30 minutes were:

a) Hello World program

b) A program that reads name and date of birth of a person and get the number of days he has lived

c) A program that accepts as input 10 numbers and outputs their sum and average.

\section{Experiment Execution Protocol}

Our application captures an image every four seconds; to classify the image we take the amplitude value of emotions with greater intensity of the EEG signals and store it in a specific directory in the appropriate folder according to the student emotion. The directory contains four folders, one for each emotion (Engagement, Boredom, Frustration and Excitement). Additionally we recorded the name of the image and the amplitude values of the signals in a text file.

This process is performed individually with each of the eight participants, during the morning using the Master of Science laboratory from the Instituto Tecnológico de Culiacán, registering an average of 450 photographs by participant.

\section{Debugging of Facial Expressions Database}

We eliminate the photographs that had some type of facial obstruction, closed eyes, or have no frontal posture. The images were cut massively to reduce the margin of the image and emphasize the subject's face. At the end we had a total of 730 photographs. Figure 4 shows some photographs stored in our corpus.

\section{$5 \quad$ Evaluation, Results, and Conclusions}

Next to the creation of the corpus, we evaluate it with an application for emotion recognition for facial expressions using the technique local binary patterns (LBP). The recognizer working with 118 photographs had an accuracy of $86.95 \%$. Due to the positive results in the evaluation, the use of this method for the preparation of a spontaneous learning-centered facial expressions database is considered feasible. For future work, we will perform the final experiment in a highly controlled environment and with improved lighting equipment, increasing the number of subjects, and taking into account the emotion meditation. 


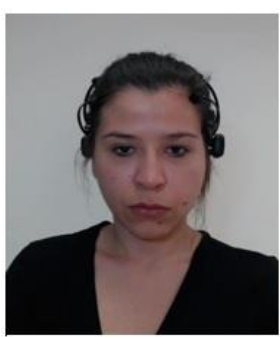

Engagement

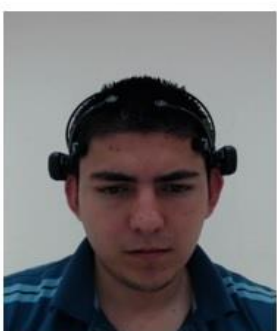

Frustration

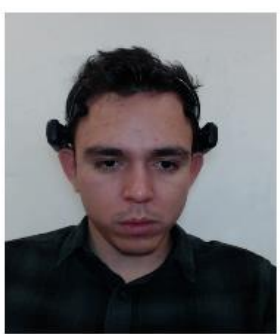

Boredom

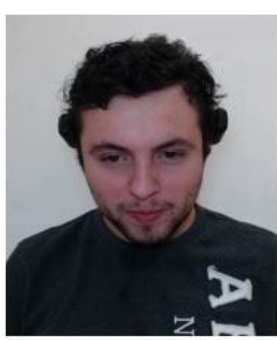

Excitement

Fig. 4. Examples of facial expressions stored in the corpus.

\section{References}

1. Barrón-Estrada, M. L., Zatarain-Cabada, R., Beltrán, J. A., Cibrian, F. L., HernándezPérez, Y.: An Intelligent and Affective Tutoring System within a Social Network for Learning Mathematics. Lecture Notes in Computer Science, 7637, pp. 651-661 (2012)

2. Hickey, T. J., Tarimo, W. T.: The affective tutor. Journal of Computing Sciences in Colleges, 29(6), pp. 50-56 (2014)

3. Petrovica, S., Pudane, M.: Simulation of Affective Student-Tutor Interaction for Affective Tutoring Systems: Design of Knowledge Structure. International Journal of Education and Learning Systems, 1 (2016)

4. Bosch, N., Mello, S. D., Hall, F., Baker, R., Shute, V., Wang, L.: Automatic Detection of Learning - Centered Affective States in the Wild. In: Proc. 20th Int. Conf. Intell. User Interfaces, pp. 379-388 (2015)

5. Anitha, C., Venkatesha, M., Adiga, B.: A survey on facial expression databases. International Journal of Engineering Science and Technology, 2(10), pp. 5158-5174 (2010)

6. Koelstra, S., Muhl, C., Soleymani, M., Yazdani, A., Ebrahimi, T., Pun, T., Nijholt, A., Patras, I.: DEAP: A Database for Emotion Analysis ;Using Physiological Signals. IEEE Trans. Affect. Comput., 3(1), pp. 18-31 (2012)

7. Wang, S., Liu, Z., Lv, S., Lv, Y., Wu, G., Peng, P., Chen, F., Wang, X.: A natural visible and infrared facial expression database for expression recognition and emotion inference. IEEE Trans. Multimed., 12(7), pp. 682-691 (2010)

8. Bartlett, M. S., Littlewort, G., Frank, M., Lainscsek, C., Fasel, I., Movellan, J.: Recognizing facial expression: Machine learning and application to spontaneous behavior. Proc. IEEE Comput. Soc. Conf. Comput. Vis. Pattern Recognit., 2, pp. 568573 (2005) 
María Lucía Barrón-Estrada, Ramón Zatarain-Cabada, Bianca Giovanna Aispuro-Medina, et al.

9. Lucey, P., Cohn, J. F., Kanade, T., Saragih, J., Ambadar, Z., Matthews, I.: The extended Cohn-Kanade dataset $(\mathrm{CK}+)$ : A complete dataset for action unit and emotion-specified expression. IEEE Comput. Soc. Conf. Comput. Vis. Pattern Recognit. - Work, CVPRW 2010, no.July, pp. 94-101 (2010)

10. Cohn, J. F., Ambadar, Z., Ekman, P.: Observer-based measurement of facial expression with the Facial Action Coding System. Handb. Emot. Elicitation Assessment, pp. 203221 (2007) 